\title{
Stochastic Processes and Dispersion of Configurations of Linked Events
}

\author{
Chan-Mou Tchen
}

\begin{abstract}
By the use of a function for the transition probability, as introduced by Kolmogoroff in diffusion problems with continuous motion (the so-called continuous stochastic processes), the dispersion of the configurations of linked events is studied. A long-chain molecule of irregular configurations, representing configurations of events linked in a time sequence, is chosen as a model. Attention is given in particular to the inversion of the dispersion law of stochastic processes, and to the interactions between nearest neighbors. A very elementary application is given to the perturbance on the distribution function for the configurations of a chain molecule, as a result of interactions by other neighbor chains.
\end{abstract}

\section{Introduction}

We can distinguish problems concerning the motion of particles as occurring in physics and mechanics into two types: (1) problems relating to completely determinate systems; (2) problems relating to irregular systems.

In the problems of the first type, the motion of the particles is controlled by certain known equations, so that its whole course can be derived from prescribed initial conditions. In the problems of the second type, on the contrary, the motion is not exactly known, even if the initial conditions were given. Such cases are found when particles are subjected, for instance, to irregular influences of mutual impacts and thermal agitations. In such cases it is only possible to calculate a distribution of the chances for the occurrence of particular positions of the particles at a later instant, assuming that certain initial conditions have been given. Such systems can be called "stochastic processes" or "irregular processes."

It is assumed that at a given instant $s^{\prime}$ there are many particles with positions between $r^{\prime}{ }_{i}$ and $r^{\prime}{ }_{i}+d r^{\prime}{ }_{i}$ (in a volume element $d r^{\prime}{ }_{1} d r^{\prime}{ }_{2} d r^{\prime}{ }_{3}$ ), where $r^{\prime}$ is a vector with components $r_{1}^{\prime}, r_{2}^{\prime}, r_{3}^{\prime}$, and $i=1,2,3$. Let the density distribution of these particles be

Let

$$
W\left(s^{\prime}, r^{\prime}\right) d r^{\prime}{ }_{1} d r_{2}^{\prime} d r^{\prime}{ }_{3} .
$$

$$
p\left(s^{\prime}, r^{\prime} ; s, r\right) d r_{1} d r_{2} d r_{3}
$$

be the probability for a particle that at the instant $s^{\prime}$ started from the region between $r^{\prime}{ }_{i}$ and $r^{\prime}{ }_{i}+d r^{\prime}{ }_{i}$ to arrive in the region between $r_{i}$ and $r_{i}+d r_{i}$ at the instant $s$. The transition probability function $p$ will be called the dispersion function, since it controls the dispersion process of particles as due to irregular motion.

The above general description of the stochastic processes has been studied by Kolmogoroff (1931, 1933) in the diffusion of particles by continuous motion. The present author has extended the theory in such a way that it can be applied to the dispersion of the elements of volume of liquid in a turbulent motion (see Tchen, 1944). In this paper, we will discuss certain general properties of the dispersion function and other derived functions, and will show how such considerations of stochastic processes are relevant to the description of the configurations of long chain molecules.

The particles (atoms, monomer units, etc.) of a long chain molecule are linked in a random arrangement. This may be considered as a spatial model of the configurations of events linked in a time sequence. Thus the variable $s$ in equation 1, which determines the time sequence of the occurrence of the position $r$ is, in the model, equal to the number of links separating the given particle from the origin of the chain. For the sake of abbreviation $s$ will be called "link-number". We consider a great number of chains of identical overal structure, but which at a given instant give configurations independent from each other. A chain will be called $O A_{s}, A_{s}$, the particles $A_{s^{\prime}}, A_{s}$ being labeled by their respective link-numbers $s^{\prime}$ and $s$. A system of coordinates with fixed directions is attached to the origin $O$ of the chain. The position of the particle $A_{s^{\prime}}$ in the system of coordinates is given by the vector $O A_{s^{\prime}}=r^{\prime}$ with components $r_{1}^{\prime}, r_{2}^{\prime}, r_{3}^{\prime}$. It is assumed that at a given instant there are many chains that have their $A_{s^{\prime}}$-particle in the positions between $r_{i}^{\prime}$ and $r_{i}^{\prime}+d r^{\prime}{ }_{i}$. Let

$$
W\left(s^{\prime}, r^{\prime}\right) d r_{1}^{\prime} d r_{2}^{\prime} d r^{\prime}
$$

be the probability for the particle $A_{s}{ }^{\prime}$ to be situated in the region between $r^{\prime}{ }_{i}$ and $r^{\prime}{ }_{i}+d r^{\prime}{ }_{i}$.

In an analogous way we can define

$$
W(s, r) d r_{1} d r_{2} d r_{3}
$$

as the probability for the particle $A_{s}$ to be situated in the region between $r_{i}$ and $r_{i}+d r_{i}$. The transition from the state $\left(s^{\prime}, r^{\prime}\right)$ to the state $(s, r)$ can be defined by a transition probability

$$
p\left(s^{\prime}, r^{\prime} ; s, r\right) d r_{1} d r_{2} d r_{3} .
$$

It is the probability for a particle $A_{s}$ to be situated in the region between $r_{i}$ and $r_{i}+d r_{i}$, being given that a preceding particle $A_{s^{\prime}}$ was situated in the region 
between $r_{i}^{\prime}$ and $\mathrm{r}_{i}{ }_{i}+d r^{\prime}{ }_{i}$. The function $p$ has a complete formal analogy with (2), and in the present application will be called the dispersion function of the linked particles.

It is to be noticed that the introduction of a discontinuous link-number $s$ in the above picture is a matter of convenience. In fact the picture can very well be generalized to a continuous chain, where we have to do with "elements of chain" instead of "linked particles". Then the position $s$ of the element in the chain can be determined by its contour length from the origin, which length may be expressed dimensionlessly, for instance, in terms of a "correlation length". (About the correlation length, see Tchen 1947).

Some properties of the dispersion function and its differential equations will be discussed briefly for the three-dimensional case in section II, by following the one-dimensional procedure of section 5 of Tchen (1944) and section 1.3 of Tchen (1947) in the study of diffusion problems.

It is to be remarked that the above dispersion function refers to increasing $s$. However, the inverse problem might also be formulated: we could ask to find from which preceding distribution an observed distribution may have originated. If $s$ refers to the time variable in the dispersion processes of discrete systems, this will mean to determine the past from the future distribution. Such an inversion of a law of nature presents special difficulties and will be discussed in section III. (The notion of inversion of a law of nature has been introduced by Schrödinger 1931). As an application, we may consider cases where the distribution of the positions at the final instant can be perturbed by interactions between the distributions of positions at the earlier instants. In order to calculate such interactions, it will be necessary to know the distributions of positions at the initial instants, being given the distribution of positions at the final instant. Such a transition from the final state to the initial state is an inversion of the dispersion processes.

In order to study further details in the mechanism of interactions, in particular when we consider two particles as nearest neighbors, or when we consider the interaction between the far particle of a molecule (particle situated in the farthest perimeter) with surrounding molecules, it will be necessary to formulate the nearest neighbor and farthest neighbor probability functions. Those functions will be studied in section IV. While the derivations of the foregoing functions and the analysis of their properties form the subject matter of this paper, elucidating the mathematical basis of the dispersion of chain configurations, a very elementary application is given finally in section $\mathrm{V}$, in order to illustrate as an example, the perturbance on the distribution as a result of interactions. This at the same time will furnish an opportunity of pointing out what reasonings are underlying the mechanism of interactions, and where the difficulties arise.

\section{Properties of the Dispersion Function and Its Differential Equations}

The distribution function $W$ and the dispersion function $p$ must satisfy the relations

$$
\begin{gathered}
\int_{-\infty}^{+\infty} d r_{1}^{\prime} \ldots W\left(s^{\prime}, r^{\prime}\right)=1 ; \int_{-\infty}^{+\infty} d r_{1} \ldots W(s, r)=1 \\
\int_{-\infty}^{+\infty} d r_{1} \ldots p\left(s^{\prime}, r^{\prime} ; s, r,\right)=1
\end{gathered}
$$

The notation

$$
\int_{-\infty}^{+\infty} d r_{1} \ldots \text { represents } \int_{-\infty}^{+\infty} d r_{1} \int_{-\infty}^{+\infty} d r_{2} \int_{-\infty}^{+\infty} d r_{3}
$$

for the sake of abbreviation. Formulas in (3) express the condition of normalization, and formula (4) expresses the condition that all transitions starting out of the state $\left(s^{\prime}, r^{\prime}\right)$ must find their places in the totality of the elements $d r_{1} \ldots$, forming the available domain of the variable $r_{i} . p$ is the transition probability from a known initial state $\left(s^{\prime}, r^{\prime}\right)$ to a final state $(s, r)$. So, if the initial distribution $W\left(s^{\prime} r^{\prime}\right)$ is known, the final distribution $W(s, r)$ can be deduced from the relation

$$
W(s, r)=\int_{-\infty}^{+\infty} d r^{\prime}{ }_{1} \ldots W\left(s^{\prime}, r^{\prime}\right) p\left(s^{\prime}, r^{\prime} ; s, r\right) .
$$

In order to conform to the laws of diffusion phenomena, the dispersion function moreover must satisfy the condition (see Smoluchowski, 1913 and Chapman, 1928):

$p\left(s^{\prime}, r^{\prime} ; s, r\right)=$

$$
\int_{-\infty}^{+\infty} d r^{\prime \prime}{ }_{1} \ldots p\left(s^{\prime}, r^{\prime} ; s^{\prime \prime}, r^{\prime \prime}\right) p\left(s^{\prime \prime}, r^{\prime \prime} ; s, r\right),
$$

where $s^{\prime} \leq s^{\prime \prime} \leq s$. This equation means that the diffusion process is integrable, $i$. $e$., the process from $\left(s^{\prime}, r^{\prime}\right)$ to $(s, r)$ as controlled by the function $p\left(s^{\prime}, r^{\prime} ; s, r\right)$ can be obtained by calculating first the dispersion in the interval $s^{\prime \prime}-s^{\prime}$, and then the dispersion in the adjoining interval $s-s^{\prime \prime}, s^{\prime \prime}$ being an arbitrary intermediary variable within the interval $s-s^{\prime}$.

Under the conditions expressed by (4) and (6), the dispersion function will satisfy two partial differential equations given originally by Kolmogoroff (1931 and 1933). They have been derived by Tchen (1944) independently of Kolmogoroff's methods. In the following lines, we shall first discuss some developments into series of the dispersion function before returning to those partial differential equations. 
It is in the nature of the phenomena of motion, whether regular or irregular that we must expect

$$
\lim _{s=s^{\prime}} p\left(s^{\prime}, r^{\prime} ; s, r\right)=0 \quad \text { for } r \neq r^{\prime}
$$

i. e., when there is a finite difference between $r$ and $r^{\prime}$. Nevertheless relation (4) must remain valid. Consequently for $r=r^{\prime}$, the function $p\left(s^{\prime}, r^{\prime} ; s, r\right)$, which will be finite for $s \neq s^{\prime}$, must take necessarily an integrable infinite value, when $s$ tends indefinitely to $s^{\prime}$, so that (4) is not violated. Such properties of the $p$-function at the limit when $s$ tends to $s^{\prime}$, re- sembles to those of the Dirac operator, the $\delta$-function, which is a kind of improper function. Certain operations involving an improper function may impair the rigour. For instance, the development of $p\left(s^{\prime}, r^{\prime} ; s, r\right)$ into a Taylor series will become badly convergent or even cease to be convergent at the limit $s=s^{\prime}$. As an example let us examine the series development in the derivation of the second partial differential equation of the $p$-function by Kolmogoroff. Kolmogoroff (1931 and 1933) introduced an arbitrary function $R$, that satisfies certain boundary condition and showed that

$$
\begin{aligned}
& \int_{-\infty}^{+\infty} d r_{1} \cdots R(r) \frac{\partial p\left(s^{\prime}, r^{\prime} ; s, r\right)}{\partial s} \\
& =\lim _{\tau \rightarrow 0} \frac{1}{\tau} \int_{-\infty}^{+\infty} d r_{1} \ldots R(r) \int_{-\infty}^{+\infty} d r^{\prime \prime}{ }_{1} \ldots p\left(s^{\prime}, r^{\prime} ; s, r^{\prime \prime}\right) p\left(s, r^{\prime \prime} ; s+\tau, r\right)-\lim _{\tau \rightarrow 0} \frac{1}{\tau} \int_{-\infty}^{+\infty} d r_{1} \cdots R(r) p\left(s^{\prime}, r^{\prime} ; s, r\right) \\
& =\lim _{\tau \rightarrow 0} \frac{1}{\tau} \int_{-\infty}^{+\infty} d r^{\prime \prime}{ }_{1} \ldots \int_{-\infty}^{+\infty} d r_{1} \cdots R\left(r^{\prime \prime}\right)\left\{-\frac{\partial}{\partial r^{\prime \prime}}\left[p\left(s^{\prime}, r^{\prime} ; s, r^{\prime \prime}\right) p\left(s, r^{\prime \prime} ; s+\tau, r\right)\left(r-r^{\prime \prime}\right)_{i}\right]\right. \\
& \left.+\frac{\partial^{2}}{\partial r^{\prime \prime}{ }_{i} \partial r_{k}^{\prime \prime}}\left[p\left(s^{\prime}, r^{\prime} ; s, r^{\prime \prime}\right) p\left(s, r^{\prime \prime} ; s+\tau, r\right) \frac{\left(r-r^{\prime \prime}\right)_{i}\left(r-r^{\prime \prime}\right)_{k}}{2}\right]\right\} \text {. }
\end{aligned}
$$

The quantity in $\{.$.$\} is a development into a$ Taylor series, which may become badly convergent for values of $\tau$ approaching zero. In view of this difficulty, special care about the series development will be taken in our derivations.

In Gauss' function the parameters $s^{\prime}, r^{\prime}, s, r$ figure exclusively in the form of the differences $s-s^{\prime}, r-r^{\prime}$. In functions of a general type, however, the parameters may occur separately. Let

$$
s-s^{\prime}=\tau ; \quad r-r^{\prime}=l
$$

then we can write the dispersion function $p\left(s^{\prime}, r^{\prime} ; s, r\right)$ as a function $P$ of $\left(s^{\prime}, r^{\prime} ; \tau, l\right)$ or of $(s-\tau, r-l ; \tau, l)$ :

$$
p\left(s^{\prime}, r^{\prime} ; s, r,\right)=P\left(s^{\prime}, r^{\prime} ; \tau, l\right)=P(s-\tau, r-l ; \tau, l) .
$$

The second mode of writing is useful when we want to express that the variations of a dispersion function are much slower with respect to $s^{\prime}, r^{\prime}$ than with respect to $\tau, l$, in particular when $\tau$ is small:

$$
\partial P / \partial r_{i}^{\prime} \ll \partial P / \partial l_{i} \quad \text { for } \tau \text { small. }
$$

Hence in the development into a Taylor series that will be used in the derivations of the partial differential equations, we will use the dispersion function in the form of $p$ when $\tau$ is not small, and in the form of $P$ when $\tau$ is small. A development of $P$ into a Taylor series with respect to $r$ means a development of $p\left(s^{\prime}, r^{\prime} ; s, r\right)$ into a series proceeding simultaneously with respect to $r^{\prime}$ and to $r$, with equal increments of both variables.

With the use of the dispersion function, the moments or the mean values of the displacement and of its higher powers can be definied as follows:

$$
\left.\begin{array}{c}
\overparen{l_{i}}=\int_{-\infty}^{+\infty} d l_{1} \ldots l_{i} P(s, r ; \tau, l) \\
\overparen{l_{i} l_{k}}=\int_{-\infty}^{+\infty} d l_{1} \ldots l_{i} l_{k} P(s, r ; \tau, l)
\end{array}\right\}
$$

Following the assumptions of Kolmogoroff, moments of higher orders are negligible and the ratios $\Gamma_{i} / \tau, \widehat{l_{i} l_{k}} / 2 \tau$ tend to finite constant values at the limit $\tau \rightarrow 0$. However, we can make, as was done in Tchen (1947), a broader assumption: we assume that they tend to finite constant values for small values of $\tau$ but still exceeding the correlation measure, which was studied in detail there.

In order to derive the partial differential equations for $p$, we start from the integral equation (6). We suppose that $\tau=s^{\prime \prime}-s^{\prime}$ be a small quantity, and write $l=r^{\prime \prime}-r^{\prime} \quad{ }^{1} \quad$ If $s-s^{\prime}$ is not particularly small, neither will $s-s^{\prime \prime}$ be small. So we can develop

$$
p\left(s^{\prime \prime}, r^{\prime \prime} ; s, r\right)=p\left(s^{\prime \prime}, r^{\prime}+l ; s, r\right)
$$

into a Taylor series in powers of $l$ by keeping the dispersion function in the form of $p$. Substituting this series into (6), applying (4) and (7), and let $s^{\prime \prime}$ tend to $\mathrm{s}^{\prime}$, we obtain

$$
\frac{\partial p\left(s^{\prime}, r^{\prime} ; s, r\right)}{\partial s^{\prime}}=-\frac{\Gamma_{i}}{\tau}\left(s^{\prime}, r^{\prime}\right) \frac{\partial p}{\partial r_{i}^{\prime}}+\frac{\overparen{l_{i} l_{k}}}{2 \tau} \frac{\partial^{2} p}{\partial r_{i}^{\prime} \partial r_{k}^{\prime}} .
$$

$1 \tau$ and $l$ are intervals of the arguments of the $p$-function. For instance, if the function considered is $p\left(s^{\prime}, r^{\prime} ; s^{\prime \prime}, r^{\prime \prime}\right)$, such as in $(6)$, then $\tau=s^{\prime \prime}-s^{\prime}, l=r^{\prime \prime}-r^{\prime}$. 
Here, and in the following, a summation will be understood whenever an index occurs twice.

In the second place let us suppose that $\tau=s-s^{\prime \prime}$ is a small quantity. When we put $l=r-r^{\prime \prime}$, we may write (6) in the form

$$
\begin{aligned}
& p\left(s^{\prime}, r^{\prime} ; s, r\right)= \\
& \quad \int_{-\infty}^{+\infty} d l_{1} \ldots p\left(s^{\prime}, r^{\prime} ; s^{\prime \prime}, r-l\right) P\left(s^{\prime \prime}, r-l ; \tau, l\right) .
\end{aligned}
$$

As $\varepsilon^{\prime \prime}-s^{\prime}$ is not small, we may develop $p\left(s^{\prime}, r^{\prime} ; s^{\prime \prime}, r-l\right) P\left(s^{\prime \prime}, r-l ; \tau, l\right)$ into a Taylor series. Substituting this series into (6a), we obtain after integration with respect to $d l$ and applying (4) and (7)

$$
\begin{aligned}
p\left(s^{\prime}, r^{\prime} ; s, r\right)-p\left(s^{\prime}, r^{\prime} ; s^{\prime \prime}, r\right)= & -\frac{\partial}{\partial r_{i}}\left[l_{i}\left(s^{\prime \prime}, r\right) p\right]+ \\
& \frac{\partial^{2}}{\partial r_{i} r_{k}}\left[\frac{l_{i} l_{k}}{2} p\right] .
\end{aligned}
$$

By decreasing indefinitely $\tau$, we obtain

$$
\frac{\partial p\left(s^{\prime}, r^{\prime} ; s, r\right)}{\partial s}=-\frac{\partial}{\partial r_{i}}\left[\frac{\Gamma_{i}}{\tau}(s, r) p\right]+\frac{\partial^{2}}{\partial r_{i} \partial r_{k}}\left[\frac{\overparen{l_{i} l_{k}}}{2 \tau} p\right] .
$$

Equations (8) and (9) are the partial differential equations for the changes of $p$ with respect to $s^{\prime}$ and $s$, respectively.

A partial differential equation $W$ can also be derived by an analogous procedure as for $p$. We start from (5), which can be written as follows

$$
W(s, r)=\int_{-\infty}^{+\infty} d l_{1} \ldots W(s-\tau, r-l) P(s-\tau, r-l ; \tau, l),
$$

with $\tau=s-s^{\prime}, l=r-r^{\prime}$. The integrand can be developed into series, and by using the assumptions of Kolmogoroff, we obtain at the limit $\tau \rightarrow 0$,

$$
\frac{\partial W}{\partial t}=\frac{\partial}{\partial r_{i}}\left(W \frac{\Gamma_{i}}{\tau}\right)+\frac{\partial^{2}}{\partial r_{i} \partial r_{k}}\left(W \frac{\overparen{l}_{i} l_{k}}{2 \tau}\right) .
$$

The equation in this form is called the FokkerPlanck equation. (See Burgers 1941, Fokker 1914, Planck 1917 and Tchen 1944.)

\section{Inversion of a dispersive phenomenon}

Being given relation (5), we seek a function $p^{*}\left(s^{\prime}, r^{\prime} ; s, r\right)$ such that for every pair of functions $W\left(s^{\prime}, r^{\prime}\right), W(s, r)$ connected by relation (5), we have the following relation:

$$
W\left(s^{\prime}, r^{\prime}\right)=\int_{-\infty}^{+\infty} d r_{1} \cdots W(s, r) p^{*}\left(s^{\prime}, r^{\prime} ; s, r\right) .
$$

If such a function is found, then $p^{*}$ is called the inverse dispersion function. Now, in general, it is probable that such an inverse function will not always be positive. As an example let us take a discrete system in which the integral is replaced by a sum:

$$
\bar{W}_{i}=\sum_{j} W_{j} p_{i j} \quad i, j=1,2, \cdots N,
$$

with the conditions

$$
\sum_{i} p_{i j}=1 \quad \text { for all values of } j,
$$

and

$$
p_{i j} \geq 0 \text {. }
$$

We can solve this system of equations for the variables $W_{j}$ when $\bar{W}_{i}$ are given. Let $D$ be the determinant of the $p_{i s}$

$$
D=\left|\begin{array}{llll}
p_{11} & p_{12} & \cdots & p_{1 N} \\
p_{21} & & & \\
p_{31} & & & \\
\vdots & & & \\
p_{N 1} & \cdots & & p_{N N}
\end{array}\right|
$$

Let us denote by $D_{i j}$ its cofactor. Then we have

$$
W_{j}=\sum_{i} \frac{D_{i j}}{D} \bar{W}_{i}
$$

so that for every arbitrary distribution of the $W$ 's the relation is inverse.

Now there exists the known relation

$$
\sum_{i} \frac{D_{i j} p_{i k}}{D}=\left\langle\begin{array}{l}
1, \text { if } j=k \\
0, \text { if } j \neq k
\end{array}\right.
$$

As $p_{i k} \geq 0$ for all $i$ and $k$, it is not possible that always

$$
\frac{D_{i j}}{D} \geq 0
$$

When negative values occur among the $D_{i j} / D$, these quantities cannot be considered as determining a probability. Thus we cannot use them to answer the question: What is the probability that a particle that is situated at $r$ at the phase $s$ (the variable $s$ may be the time), was situated at $r^{\prime}$ at a previous phase $s^{\prime}$ ?

As an example we consider a case of travels from the cities $C_{1}$ and $C_{2}$ to the cities $\overline{C_{1}}$ and $\overline{C_{2}}$, For each city of start, we can define the probability of a travel to $\overline{C_{1}}$ or $\overline{C_{2}}$ independently of the number of travelers leaving the cities of start, e. g., 


$\begin{array}{lll}\text { from } C_{1}: & 0.6 \text { to } \bar{C}_{1}, & 0.4 \text { to } \bar{C}_{2}, \\ \text { from } C_{2}: & 0.4 \text { to } \overline{C_{1}}, & 0.6 \text { to } \overline{C_{2}} .\end{array}$

Thus relation (12) can be written into the equations

$$
\left.\begin{array}{l}
\overline{W_{1}}=0.6 W_{1}+0.4 W_{2} \\
\overline{W_{2}}=0.4 W_{1}+0.6 W_{2}
\end{array}\right\},
$$

where $W_{1}, W_{2}$ are numbers of travelers in cities of start $C_{1}$ and $C_{2}$, and $\bar{W}_{1} \bar{W}_{2}$ are numbers of travelers in cities of destination $\overline{C_{1}}$ and $\overline{C_{2}}$.

Inversely we can solve the system of (15) for the variables $W_{1}, W_{2}$ in terms of $\overline{W_{1}}, \bar{W}_{2}$. We have

$$
\left.\begin{array}{l}
W_{1}=3 \overline{W_{1}}-2 \overline{W_{2}} \\
W_{2}=-2 \overline{W_{1}}+3 \overline{W_{2}}
\end{array}\right\}
$$

It is seen that $p^{*}$ (coefficients of $\overline{W_{1}}$ and $\overline{W_{2}}$ ) presents also negative values and hence cannot be considered as determining a probability.

The dispersion function $p$ as governed by the differential equation (9) has the task of bringing the distribution at a certain initial phase $s^{\prime}$ into a broader distribution at a later phase $s$. So, on the other hand, in the inverse problem, we must expect a narrowing of the distribution. However, such an inverse dispersion in the narrowing transition of distributions cannot exist without having certain negative values either in the inverse dispersion function or in the distribution function. From the above considerations we can conclude that in a stochastic world, we can find a law of transition (dispersion function) that tells us about a distribution of the future from a distribution of the past. However, for someone living in the inverse world where all events present in the wrong way, it will not be possible to find an inverse law of transition (inverse dispersion function) that tells about a distribution of the past from a distribution of the future, if the law of transition and of distribution are to be positive.

In order to answer in the inverse problem the question: what is the preceding distribution from which an observed distribution has originated, we may write:

for the case of travels:

$$
\begin{aligned}
& W_{1}=0.6 \frac{W_{1}}{\overline{W_{1}}} \overline{W_{1}}+0.4 \frac{W_{1}}{\overline{W_{2}}} \overline{W_{2}}, \\
& W_{2}=0.4 \frac{W_{2}}{\overline{W_{1}}} \overline{W_{1}}+0.6 \frac{W_{2}}{\overline{W_{2}}} \overline{W_{2}},
\end{aligned}
$$

for a discontinuous system in general:

$$
W_{j}=\sum_{i} p_{i j}^{\prime} \overline{W_{i}}
$$

where

$$
p^{\prime}{ }_{i j}=p_{i j} \frac{W_{j}}{\bar{W}_{i}}
$$

and for a continuous system:

$$
W\left(s^{\prime}, r^{\prime}\right)=\int_{-\infty}^{+\infty} d r_{1} \ldots W(s, r) p^{\prime}\left(s^{\prime}, r^{\prime} ; s, r\right),
$$

where

$$
p^{\prime}\left(s^{\prime}, r^{\prime} ; s, r\right)=p\left(s^{\prime}, r^{\prime} ; s, r\right) \frac{W\left(s^{\prime}, r^{\prime}\right)}{W(s, r)} .
$$

We may consider $p^{\prime}$ as the probability for a particle to have started from $d r^{\prime}{ }_{i}$ at the phase $s^{\prime}$, being given its position in $d r_{i}$ at the phase $s$. In a certain sense, the function $p^{\prime}$ plays the role of a kind of inverse function of the dispersion function $p$. However, $p^{\prime}$ is dependent upon the distribution really present, and has no independent meaning such as was the case with $p . p^{\prime}$ will be called retrograde dispersion function. By eliminating $p$ from (5) and (17) it is seen that $p^{\prime}$ satisfies the condition of normalization

$$
\int_{-\infty}^{+\infty} d r_{1}^{\prime} \ldots p^{\prime}\left(s^{\prime}, r^{\prime} ; s, r\right)=1 .
$$

Let us now specify the above considerations of retrograde dispersion for a chain molecule in the description of the intramolecular interaction. Consider a chain $A_{o} A_{s^{\prime}} A_{s}$ with origin at $A_{o}$. Being given the position $r^{\prime}$ of $A_{s^{\prime}}$, the probability for the particle $A_{s}$ to be situated between $r_{i}$ and $r_{i}+d r_{i}$ is by definition

$$
p^{\prime}\left(s^{\prime}, r^{\prime} ; s, r\right) d r_{1} d r_{2} d r_{3} .
$$

Since the chain is symmetrical, we can take the form

$$
p^{\prime}\left(s^{\prime}, r^{\prime} ; s, r\right)=W\left(s-s^{\prime}, r-r^{\prime}\right),
$$

depending upon the difference $s-s^{\prime}$ and $r-r^{\prime}$. Hence (17) becomes

$p^{\prime}\left(s^{\prime}, r^{\prime} ; s, r\right)=W\left(s-s^{\prime}, r-r^{\prime}\right) W\left(s^{\prime}, r^{\prime}\right) / W(s, r)$

This relation means that the probability $p^{\prime}\left(s^{\prime}, r^{\prime} ; s, r\right) d r^{\prime}{ }_{1} d r^{\prime}{ }_{2} d r^{\prime}{ }_{3}$ for the particle $A_{s^{\prime}}$ to be situated in $d r^{\prime}{ }_{1} d r_{2}{ }_{2} d r^{\prime}{ }_{3}$, being given the position $r_{i}$ of the particle $A_{s}$ from $A_{o}$, is equal to the product of the following two probabilities:

(a) The probability $W\left(s^{\prime}, r^{\prime}\right) d r^{\prime}{ }_{1} d r^{\prime}{ }_{2} d r^{\prime}{ }_{3}$ for the particle $A_{s^{\prime}}$ to be situated in the region between $r^{\prime}{ }_{i}$ and $r^{\prime}{ }_{i}+d r^{\prime}{ }_{i}$ from $A_{0}$.

(b) The probability $W\left(s-s^{\prime}, r-r^{\prime}\right) d r_{1}^{\prime} d r_{2}{ }_{2} d r^{\prime}{ }_{3}$ for the particle $A_{s^{\prime}}$ to be situated in the region between $r_{i}-r_{i}^{\prime}$ and $r_{i}-\left(r_{i}^{\prime}+d r_{i}{ }_{i}\right)$ from $A_{s}$, with $A_{o} A_{s}=r_{i}$. 
This latter probability, argued from a symmetrical inversion of the chain, assures the condition that the particle $A_{o}$ is situated at $r_{i}$ from $A_{s}$. By taking the product of (a) by (b), we have

$$
\begin{aligned}
& p^{\prime}\left(s^{\prime}, r^{\prime} ; s, r\right) d r^{\prime}{ }_{1} \ldots= \\
& \text { constant } W\left(s-s^{\prime}, r-r^{\prime}\right) W\left(s^{\prime}, r^{\prime}\right) d r^{\prime}{ }_{1} \ldots
\end{aligned}
$$

This expression becomes identical to (20) after determination of the constant by the condition of normalization (18).

In the study of intramolecular interactions of long chain molecules, the above retrograde dispersion function is in general not sufficient. We have to know, in general, the probability of the distance between an arbitrary pair of particles, say $A_{j}$ and $A_{l}$ which none of them are ends of the chain. In the following we shall show how such a more complicated retrograde dispersion function can be constructed on the basis of the simple one obtained in (20).

Let us now consider a chain $A_{o} A_{j} A_{l} A_{s}$. Being given the distance $r_{i}$ of the particle $A_{s}$ from the particle $A_{o}$, we ask the probability

$$
p^{\prime}\left(A_{j} A_{l}, h ; s, r\right) d h_{1} \ldots
$$

for the particle $A_{l}$ to be situated in the position between $h_{i}$ and $h_{i}+d h_{i}$ from $A_{j}$. It is the product of the following probabilities:

(a) Both ends $j$ and $l$ must be subjected to certain conditions, namely $A_{j}$ must be in the position between $\lambda_{i}-h_{i}$ and $\lambda_{i}+d \lambda_{i}-h_{i}$ from $A_{o}$ with probability

$$
W(j, \lambda-h) d \lambda_{1} \ldots
$$

(b) $A_{l}$ must be in the position between $r_{i}-\lambda_{i}$ and $r_{i}-\lambda_{i}-d \lambda_{i}$ from $A_{s}$ with the probability

$$
W(s-l, r-\lambda) d \lambda_{1} \ldots .
$$

The product of the above probabilities integrated with respect to $\lambda$ gives

$$
\begin{aligned}
& p^{\prime}\left(A_{j} A_{l}, h ; s, r\right) \\
& =\text { const. } \int_{-\infty}^{+\infty} d \lambda_{1} \ldots W(j, \lambda-h) W(s-l, r-\lambda), \\
& =\int_{-\infty}^{+\infty} d \lambda_{1} \ldots \frac{W(l-j, h)}{W(s, r)} p(l-j, h ; l, \lambda) p(l, \lambda ; s, r),
\end{aligned}
$$

using (17) and (19),

$$
=p(l-j, h ; s, r) \frac{W(l-j, h)}{W(s, r)} \text {, using (6). }
$$

According to (17), the right-hand member is $p^{\prime}(l-j, h ; s, r)$. Hence

$$
p^{\prime}\left(A_{j} A_{l}, h ; s, r\right)=p^{\prime}(l-j, h ; s, r) .
$$

This shows that the retrograde dispersion function for an intermediary pair of particles $A_{l}$ and $A_{j}$ is equal to the retrograde dispersion function for the end pair of particles $A_{l-y}$ and $A_{0}$. Thus in the following section, by studying the nearest neighborhood dispersion function, we can consider only the latter dispersion function for the end pair of particles, by putting $s=l-j$ and $h=r^{\prime}$.

It will be useful to calculate the $p^{\prime}$-function of (22) from (21) for a $W$-function of the Gaussian type:

$$
W(s, r) d r_{1} \ldots=\left(\frac{\kappa^{2}}{\pi}\right)^{3 / 2} e^{-\kappa^{2} r^{2}} d r_{1} \ldots
$$

with $\kappa^{2}=\left(3 / 2 b^{2}\right)(1 / s)$, and $b=$ length of one link. The use of (19) and of polar coordinates transforms (21) into the following formula after integration with respect to the polar angles

$$
\begin{aligned}
p^{\prime}\left(A_{1} A_{b}, h ; s, r\right) 4 \pi h^{2} d h & =p^{\prime}(l-j, h ; s, r) 4 \pi h^{2} d h \\
& =\frac{\mu}{\sqrt{\pi}} \frac{h}{a}\left[e^{-\mu^{2}(h-a)^{2}}-e^{-\mu^{2}(h+a)^{2}}\right],
\end{aligned}
$$

where

$$
\mu^{2}=\frac{3}{2 b^{2}} \frac{s}{(l-j)(s-l+j)} ; a=r(l-j) / s .
$$

This formula is an extended form of a formula obtained by Kuhn, Künzle and Katchalsky (1949) for the special case of $j=0$.

\section{Nearest Neighbor Probabilities}

In the study of the mechanism of interaction, we have to know not only the distribution of the distance between two arbitrary particles, but also often such a distribution as they become nearest neighbors. For discrete particles that are totally independent from each other, Herz (1909) has obtained a formula for the nearest neighbor distribution functions in terms of the distribution function. Applications have been made by Jaffé (1940) to the study of interactions of charged particles. Applications to linked particles, such as is the case with a chain molecule, has been made by Simha (1948), in connection with the problem of volume effect. However, such a direct application of Herz's formula to the density distribution of linked particles yields only an approximate result. In fact the different particles on the chain behave differently as nearest neighbors, according to their order of linkage, in such a way that the pure randomness of the particles, lying in the indifference of order, will be distorted. It is the purpose of the following lines to study the nearest neighbor probabilities for such mutually 
dependent particles. Herz's method will be extended to the calculations of the nearest neighbor function of the retrograde dispersion function.

Consider again a chain $O A_{s^{\prime}} A_{s}\left(s>s^{\prime}\right)$ with origin of coordinates at $O$, the case of spherical symmetry being considered. Being given the scalar distance $r$ of the particle $A_{s}$ from the origin, the probability for the particle $A_{s^{\prime}}$ to be situated in the region between $r^{\prime}$ and $r^{\prime}+d r^{\prime}$ is

$$
p^{\prime}\left(s^{\prime}, r^{\prime} ; s, r\right) 4 \pi r^{\prime 2} d r^{\prime},
$$

where $p^{\prime}$ is the retrograde dispersion function. Its nearest neighbor function will be designated by $p^{\prime}{ }_{1}$. It is the propability for the particle $A_{s^{\prime}}$ to be situated in the region between $\mathrm{r}^{\prime}$ and $r^{\prime}+d r^{\prime}$, as a nearest neighbor to the particle $O$, being given the distance $O A_{s}=r$ of the particle $A_{s}$. It can be calculated by taking the product of the following two probabilities.

(a) the probability that the particle $A_{s^{\prime}}$ is situated at a distance between $r^{\prime}$ and $r^{\prime}+d r^{\prime}$ from $O$, being given the distance $O A_{s}=r$ for the particle $A_{s}$;

(b) the probability that no nearest neighbor is situated in the region between 0 and $r^{\prime}$.

The probability indicated in (a) is given by (25), but owing to the fact that $A_{s^{\prime}}$ cannot be situated in the region between 0 and $r^{\prime}$, the probability in (a) will be increased to the value

$$
p^{\prime} 4 \pi{r^{\prime}}^{2} d r^{\prime} /\left(1-\int_{0}^{r^{\prime}} d r^{\prime} 4 \pi{r^{\prime}}^{2} p^{\prime}\right) .
$$

On the other hand, the probability of finding any one of the $s$ particles as a nearest neighbor to the particle $O$ in the region $d r^{\prime}$ is

$$
\sum_{s^{\prime}=1}^{s} p_{1}^{\prime}\left(s^{\prime}, r^{\prime} ; s, r\right) 4 \pi r^{\prime 2} d r^{\prime}
$$

say denoted by $\varpi 4 \pi r^{\prime 2} d r^{\prime}$ and the probability that no nearest neighbor is situated in the region between 0 and $r^{\prime}$ is

$$
1-\int_{0}^{r^{\prime}} d r^{\prime} 4 \pi{r^{\prime}}^{2} \varpi
$$

Hence by taking the product of the probabilities in (26) and (27a) and using (27), we obtain the following integral equation for $\varpi$

$$
\begin{aligned}
& \varpi 4 \pi{r^{\prime}}^{2} d r^{\prime}= \\
& {\left[1-\int_{0}^{r^{\prime}} d r^{\prime} 4 \pi{r^{\prime}}^{2} \varpi\right] \cdot \sum_{s^{\prime}=1}^{s} \frac{p^{\prime} 4 \pi{r^{\prime}}^{2} d r^{\prime}}{1-\int_{0}^{r^{\prime}} d r^{\prime} 4 \pi r^{\prime 2} p^{\prime}}}
\end{aligned}
$$

In order to simplify the writing, we put ${ }^{2}$

$$
\begin{aligned}
T & =\sum_{s^{\prime}=1}^{s} \frac{p^{\prime} 4 \pi r^{\prime 2}}{1-\int_{0}^{r^{\prime}} d r^{\prime} 4 \pi{r^{\prime}}^{2} p^{\prime}} \\
& =-\frac{d}{d r^{\prime}} \sum_{s^{\prime}=1}^{s} \ln (1-Q)=-\sum_{s^{\prime}=1}^{s} \dot{R} \\
Q & =\int_{0}^{r^{\prime}} d r^{\prime} 4 \pi{r^{\prime}}^{2} p^{\prime} ; \quad R=\ln (1-Q) ;
\end{aligned}
$$

then (28) can be written in the simpler form

$$
\varpi 4 \pi{r^{\prime}}^{2} d r^{\prime}=T\left(1-\int_{0}^{r^{\prime}} d r^{\prime} 4 \pi{r^{\prime}}^{2} \varpi\right) d r^{\prime}
$$

An integration gives the solution under one of the following forms:

$$
\begin{aligned}
\varpi 4 \pi{r^{\prime}}^{2} & =T \prod_{s^{\prime}=1}^{s}(1-Q) \\
& =T e^{-\int_{0}^{r^{\prime}} d r^{\prime} T .}
\end{aligned}
$$

It can be shown that $\varpi$ satisfies the condition of normalization. We write from (29a)

$$
\varpi 4 \pi r^{\prime 2}=-\sum_{s^{\prime}=1}^{s} \dot{R} e^{s^{s^{2}} R} R
$$

By integrating with respect to $r^{\prime}$, we have

$$
\int_{0}^{r^{\prime}} d r^{\prime} 4 \pi r^{\prime 2} \varpi=1-e^{\stackrel{s}{s^{\prime}=1} R} .
$$

By increasing the upper limit indefinitely, we obtain

$$
\int_{0}^{\infty} d r^{\prime} 4 \pi r^{\prime 2} \varpi=1 .
$$

This condition means that one of the $s$ particles at least must be a nearest neighbor to the particle $O$ in the totality of the domain.

The function $p^{\prime}{ }_{1}$ gives the probability of the distances between two nearest neighbor particles that are linked on a chain. If the particles are not linked and behave like gas molecules, we can define a distribution $W(r)$ of the distances $r$ between two particles, and a distribution $W_{1}(r)$ when the particles are nearest neighbors. For this latter case, the nearest neighbor distribution $W_{1}$ can be obtained

${ }^{2}\left(^{\circ}\right)$ means $\frac{d}{d r^{\prime}}$. 
from (29) by changing $p^{\prime}, p^{\prime}{ }_{1}$ into $W / s, W_{1} / s$, respectively, $\dot{W}$ and $W_{1}$ being now independent of $s^{\prime}$. So for discrete particles formula (29) degenerates to the formula of Herz (1909):

$$
W_{1}(r)=W(r)\left[1-\frac{1}{s} \int_{0}^{r} d r 4 \pi r^{2} W\right]^{s-1},
$$

where $s$ is the number of such independent particles.

In the study of intramolecular interactions, $i . e$., the interactions between the different parts of one giant chain, the configurations of any particle of the chain are wholly controlled by the dispersion functions $p, p^{\prime}$, and $p^{\prime}{ }_{1}$ as studied above. On the other hand, in the study of intermolecular interactions, $i$.e., the interactions between two particles belonging to two different chains, we might ask: what is the probability for the particle $A_{s^{\prime}}$, belonging to one chain, say chain $B$, to be situated at a distance between $r^{\prime}$ and $r^{\prime}+d r^{\prime}$ from the origin $O$, at which distance it becomes the farthest one of all particles of $B$, and therefore becomes the most exposed to interactions with surrounding chains? Such probabilities, as will be indicated by the subscript " 2 ", e. g. $W_{2}, p^{\prime}{ }_{2}$, will be called "farthest neighbor probabilities", in contrast with nearest neighbor probabilities. The farthest neighbor probabilities can be calculated in the same way as for the nearest neighbor probabilities, except that the limits $\left(0, r^{\prime}\right)$ have to be changed into $\left(r^{\prime}, \infty\right)$. Following (29a) the result is

$p_{2}^{\prime}=\frac{p^{\prime}}{1-\int_{r^{\prime}}^{\infty} d r^{\prime} 4 \pi r^{\prime 2} p^{\prime}} e^{s^{s^{\prime}} \stackrel{s}{\Sigma} \ln \left(1-\int_{r^{\prime}}^{\infty} d r^{\prime} 4 \pi r^{\prime 2} p^{\prime}\right)}$.

\section{Intermolecular Interactions}

The intramolecular interaction, $i$. $e$. between the different parts of one giant chain molecule, is comparable to a kind of internal repulsion within the chain molecule. Therefore an expansion of the chain can be expected. On the other hand, the intermolecular interaction, $i$. $e$. between a number of separate molecules, is a kind of external repulsion exerting on every molecule, and will cause a compression of the chain. As indicated by Simha, the observed anormality of the variations of viscosity with concentration in connection with the solvent effect could point to a shrinkage of two coil-molecules on approaching each other at concentrations of a few tenths of one percent.

The perturbance on the distribution function $W(r)$, by those interactions, can be calculated on the basis of the retrograde dispersion function, nearest neighbor and farthest neighbor functions derived above. The perturbance by intermolecular interactions will be discussed here for the following very elementary case.
Let us consider the one-dimensional interaction of two chains $A$ and $B$, which are supposed to move only along the direction $O y$. We suppose that the chain $A$ is fixed at the end $A_{o}$, and that the chain $B$ is fixed at the end $B_{o}$, the point $A_{o}$ being taken as the origin of coordinates. The particle of $B$, which forms the volume hindrance to $A$, is the farthest particle of $B$, of coordinate $c$. Of course $c$ is a random variable having a certain distribution that can be calculated from the considerations given in section IV concerning the farthest neighbor probability function. This forms a difficult detail in which we shall not enter here, but assume only that we could find an effective fixed position $c$ forming a fixed obstacle to the motion of $A$. Then the distribution of any particle $A_{s^{\prime}}$ of the chain $A$ will be modified and becomes:

$$
W_{r}\left(s^{\prime}, y^{\prime}\right)=W\left(s^{\prime}, y^{\prime}\right)+W\left(s^{\prime}, 2 c-y^{\prime}\right)
$$

where $W\left(s^{\prime}, y^{\prime}\right) d y^{\prime}$ is the distribution free from obstacle, $W\left(s^{\prime}, 2 c-y^{\prime}\right) d y^{\prime}$ is the additional distribution reflected back by the obstacle, and $W_{r}\left(s^{\prime}, y^{\prime}\right) d y^{\prime}$ is the total distribution.

It is easy to verify that the total distribution satisfies the normalization condition

$$
\int_{-\infty}^{c} d y^{\prime} W_{r}\left(s^{\prime}, y^{\prime}\right)=1
$$

if the free distribution is normalized to unity:

$$
\int_{-\infty}^{+\infty} d y^{\prime} W\left(s^{\prime}, y^{\prime}\right)=1 .
$$

Let us define:

$$
\overline{y^{\prime}}=\int_{-\infty}^{+\infty} d y y^{\prime} W\left(s^{\prime}, y^{\prime}\right) ; \quad \overline{y^{\prime 2}}=\int_{-\infty}^{+\infty} d y^{\prime} y^{\prime 2} W\left(s^{\prime}, y^{\prime}\right)
$$

and

$$
\overline{y^{\prime}}=\int_{-\infty}^{c} d y^{\prime} y^{\prime} W_{r}\left(s^{\prime}, y^{\prime}\right) ; \overline{y^{\prime}{ }^{2}}=\int_{-\infty}^{c} d y^{\prime} y^{\prime 2} W_{r}\left(s^{\prime}, y^{\prime}\right)
$$

as the mean distance and the mean square distance between the particles $A_{o}, A_{s}$, of the chain $A$, in the free configuration and in the restricted configuration, respectively. With the use of (33), and for a free distribution

$$
W\left(s^{\prime}, y^{\prime}\right) d y^{\prime}=\frac{\kappa}{\sqrt{\pi}} e^{-\kappa^{2} y^{\prime 2}} d y^{\prime}
$$

with $\kappa^{2}=\left(2 s b^{2}\right)^{-1}, b=$ length of one link, it is found that:

$$
\begin{aligned}
\overline{y^{\prime}} & =0 ; \quad \overline{y^{\prime 2}}=s b^{2} \\
\overline{y_{r}^{\prime}} & =-\frac{1}{\sqrt{\pi} \kappa} e^{-\kappa^{2} c^{2}}+c(1-e r f \kappa c) ; \\
\overline{y_{r}^{\prime}{ }^{2}} & =\overline{y^{\prime 2}}+2 c \overline{y_{r}^{\prime}}
\end{aligned}
$$


The value of $\overline{y^{\prime}{ }_{r}^{2}}<\overline{y^{\prime 2}}$ indicates a compression of the chain due to intermolecular interactions.

The distributions for all particles, such as $A_{s^{\prime}}$, with $s^{\prime}<s$, are modified by intermolecular interactions in the manner indicated by (33). This is true, of course, only when the intramolecular effect is not considered, for instance the volume overlapping in one molecule must then be allowed. Otherwise the modifications of the distributions for all particles $s^{\prime}<s$ will influence the distribution $W_{r}(s, y) d y$ of the particle $A_{s}$. The study of such an intramolecular effect will involve the retrograde dispersion functions and the nearest neighbor probability functions, which determine the distribution of chances of interactions between one arbitrary pair of particles. Such complications form again a difficulty that remains to be investigated.

\section{References}

Burgers, J. M., Proc. Acad., Amsterdam, 44, 344 (1941). Chapman, S., Proc. Roy. Soc. A 119, 34 (1928).

Fokker, A. D., Ann. d. Physik (4) 43, 812 (1914); also comp. Archives Néerlandaises des Sciences Exactes, etc. (2a), 4, 379 (1918).

Herz, P., Math. Ann. 6\%, 387 (1909).

Jaffé, G., Phys. Rev. 58, 968 (1940).

Kolmogoroff, A. N., Math. Ann. 104, 415 (1931); 108, 149 (1933).

Kuhn, Künzle and Katchalsky, Helv. Chim. Acta 31, 1994 (1949).

Planck, M., Sitz. Ber. Berliner Akademie, 324 (1917).

Schrödinger, E., Sitz. Ber. Berliner Akademie, 144 (1931).

Simha, R., J. Research NBS 40, 21 (1948).

Smoluchowski, M. von, Bul. Acad. Cracovie, 424 (1913).

Tchen, C. M., Vers. Ned. Akad. v. Wetensch., Afd. Natuurk. 53, 400-410 (1944); also Meded. No. 51 (1947), Laboratorium voor Aero-en Hydrodynamica der Technische Hogeschool, Delft.

Washington, June 23, 1950. 